\title{
El concepto de objetividad en Gramsci
}

Raúl Burgos*

http://orcid.org/0000-0001-7482-1416

\begin{abstract}
El problema de si al pensamiento humano se le puede atribuir una verdad objetiva, no es un problema teórico, sino un problema práctico. Es en la práctica donde el hombre tiene que demostrar la verdad, es decir, la realidad y el poderio, la terrenalidad de su pensamiento. El litigio sobre la realidad o irrealidad de un pensamiento que se aisla de la práctica, es un problema puramente escolástico. KARL MARX, Segunda tesis sobre Feuerbach.
\end{abstract}

Introducción

El presente artículo presenta algunas conclusiones de un trabajo investigación más amplio destinado al estudio de la teoría de la hegemonía, que pone en perspectiva comparativa los abordajes de Antonio Gramsci en los Cuadernos de la Cárcel y de Ernesto Laclau y Chantal Mouffe a partir de su obra fundamental Hegemonia y estrategia socialista. El camino esbozado en esta trama condujo a comenzar por la discusión de ciertas premisas filosóficas y epistemológicas de la investigación. Este es el foco del presente texto, centrado en el concepto de objetividad. Por razones de espacio, expongo aquí el resultado provisorio sobre la posición gramsciana en relación al tema dejando para un próximo trabajo la exposición de los resultados sobre el mismo tema en la perspectiva de Laclau e Mouffe y algunas conclusiones sobre el contraste entre ambas. Dejo expreso aquí mi agradecimiento a Fortunato Maria Cacciatore, Pasquale

* Universidade Federal de Santa Catarina, Santa Catarina, Florianópolis, Brasil. 
Serra y Peter Thomas por las observaciones críticas y recomendaciones realizadas a la primera versión del trabajo.

El tema nos remite a un tópico polémico de la historia de la filosofía y en el interior de la propia tradición marxista, en la cual el debate ha sido y es intenso. Aún hoy, como en los tiempos de la elaboración gramsciana, determinadas lecturas de los conceptos involucrados se encuentran en la base de deformaciones reduccionistas en la interpretación de la dinámica societaria: las relaciones estructura/superestructura, la valoración del papel de la cultura y los medios de producción culturales, el papel de la acción política etc.

Quisiera comenzar evidenciando dos polos de un campo de tensiones teóricas en el interior de esta corriente de pensamiento sobre el tema en pauta. Por un lado, las posiciones fundamentales de Marx en la Tesis sobre Feuerbach (1845), que podemos tomar el como punto inicial o grado cero del análisis marxista sobre el tema. Aunque las Tesis deban tomarse en su integralidad - y Gramsci no deja de involucrarse con cada una de ellas -, retengamos al menos el contenido de la primera tesis, emblemática de la discusión que llevaremos a cabo en el texto:

Tesis1. El defecto fundamental de todo el materialismo anterior - incluido el de Feuerbach - es que sólo concibe las cosas, la realidad, la sensoriedad, bajo la forma de objeto o de contemplación, pero no como actividad sensorial humana, no como práctica, no de un modo subjetivo. De aquí que el lado activo fuese desarrollado por el idealismo, por oposición al materialismo, pero sólo de un modo abstracto, ya que el idealismo, naturalmente, no conoce la actividad real, sensorial, como tal. Feuerbach quiere objetos sensoriales, realmente distintos de los objetos conceptuales; pero tampoco él concibe la propia actividad humana como una actividad objetiva. Por eso, en La esencia del cristianismo sólo considera la actitud teórica como la auténticamente humana, mientras que concibe y fija la práctica sólo en su forma suciamente judaica de manifestarse. Por tanto, no comprende la importancia de la actuación "revolucionaria", "práctico-crítica" (Marx, 1987, tomo 1, p. 9).

Las Tesis tendrán importancia fundamental en la arquitectura de la filosofía de la praxis elaborada en las reflexiones carcelarias y fueron objeto particular de traducción en el Cuaderno 7 (1930). De hecho, son omnipresentes en los Cuadernos; no casualmente, según expresa Fabio Frosini (2010, p. 32), Gramsci las considera "el auténtico incunable de la 'filosofía' de Marx".

Por otro lado, como principal polo de tensiones teóricas, la intervención de Lenin en su libro Materialismo y empiriocriticismo (1908-1909), en particular su definición de materia como "realidad objetiva”. El revolucionario ruso definía la materia como "una categoría filosófica que sirve para designar la realidad objetiva 
que es dada al hombre en sus sensaciones, que es copiada, fotografiada, reflejada por nuestras sensaciones y que existe independientemente de ellas", y en este contexto indicaba que "la única propiedad de la materia con cuya admisión está ligado el materialismo filosófico es la propiedad de ser una realidad objetiva, de existir fuera de nuestra conciencia" (Lenin, 1990, pp. 120 y 251).

Esta posición leninista sirvió, en el marco de la Tercera Internacional como una sofisticada legitimación de la articulación del marxismo con el materialismo filosófico (en el fondo de carácter metafísico) ya dominante en la tradición de la Segunda Internacional en particular con la teoría sobre la constitución del conocimiento humano conocida como "teoría del reflejo", que se tornaría el eje de la teoría del conocimiento en el marxismo soviético (y para una tradición más amplia que sobrevivió a aquel, como veremos). Sin embargo, al dirigente bolchevique no le era ajeno el problema del "lado activo" de la subjetividad humana planteado por Marx en las Tesis. Así, por ejemplo, en los llamados Cuadernos filosóficos (1914-1916), Lenin (1975, p. 175) transcribe una frase de Hegel en la Lógica, la marca y anota al lado "NB (Nota Bene)": "es erróneo considerar la subjetividad y la objetividad como antítesis fijas y abstractas. Ambas son totalmente dialécticas". Y más adelante, discutiendo el tema titulado "Hegel, sobre la práctica y la objetividad del conocimiento", escribía de su propia pluma y obviamente desafiando cualquier dogma empirista: "Marx se sitúa claramente al lado de Hegel cuando introduce el criterio de la práctica en la teoría del conocimiento: véanse las Tesis sobre Feuerbach" y agrega en separado "la conciencia del hombre no sólo refleja el mundo objetivo, sino que lo crea” (Lenin, 1975, p. 200).

No obstante estas agudas observaciones de Lenin (debe tomarse en consideración, que la primera edición de los Cuadernos filosóficos es de 1933, en una Unión Soviética ya totalmente estalinizada), se consolida en el marxismo soviético un tipo de filosofía (y en sus marcos una noción de objetividad) que continuaba la tradición del materialismo metafísico de la Segunda Internacional. Gramsci verá en el libro de Nicolai Bujarin Teoria del materialismo histórico: ensayo popular de sociología marxista (1921) una condensación de la lectura mecanicista y descargará sobre él su trabajo crítico. Según afirma el filósofo alemán Wolfgang Fritz Haug, en su trabajo sobre el "manual” de Bujarin, Gramsci

[... emprendió la crítica sistemática de una formación ideológica que estaba surgiendo por entonces, pero que luego fue canonizada como Marxismo-Leninismo bajo Stalin [...]. En el contexto de la Internacional Comunista, particularmente bajo la influencia de Lenin, quien a su vez fuera influenciado por Plejanov ${ }^{1}$, lo que había resurgido había sido la filosofía - aunque,

1. Se pueden recordar las críticas de Gramsci al pensamiento "mecanicista vulgar" de Plejánov, al que 
como Gramsci percibió, en su comprensión premarxista como primera filosofía: metafísica. Desde el punto de vista de Gramsci, en lugar de ser una "metodología histórica”, el libro de texto soviético practicaba una metafísica, "aunque ingenua” [...]. Él vio que el materialismo histórico se había convertido en "la "sociología’ del materialismo metafísico" (Q 437). (Haug, 2001, pp. 70 e 72$)^{2}$.

Si la llamada lucha ideológica es, en primer lugar, una intensa disputa entre diversos modos de concebir la totalidad que denominamos "realidad" en su movimiento y mutación - es decir, una lucha por la "verdad", por la "objetividad" -, entonces una primera batalla se traba por el significado de la palabra "objetividad". De esta disputa trata este trabajo.

Veamos entonces, a grandes trazos, el tratamiento de la cuestión realizada por Gramsci en los Cuadernos de la cárcel. Para esto, creo conveniente partir de la discusión de los que considero los dos conceptos fundamentales en la configuración de la posición filosófica del autor de los Cuadernos en este tema: los conceptos de praxis y devenir.

\section{Devenir, praxis e historicidad absoluta}

\subsection{Praxis}

Si la expresión filosofía de la praxis le llega a Gramsci de Labriola (s/d, p. 50) ${ }^{3}$, que la califica como la "médula del materialismo histórico", el concepto de praxis sabemos que le viene del propio Marx, desde las Tesis sobre Feuerbach y Miseria de la Filosofía. Praxis como unidad de teoría y práctica, como acción consciente, como práctica que se funda en (y se funde con) la reflexión crítica.

considera de "escasas facultades especulativas e historiográficas" (cc, 11, \$70, t. IV, p. 349; Q 1508) pero que había sido maestro de toda una generación, incluido Lenin, que heredaría algunos de sus principales fundamentos filosóficos.

2. Las traducciones de los textos no citados en lengua española son del autor.

3. Existe una duradera disputa sobre la "paternidad" del concepto de filosofía de la praxis utilizado por Gramsci. Una corriente, lo hace remitir a la influencia del neoidealismo italiano fundamentalmente de Giovanni Gentile (en esto es fundamental la influencia de la obra de Augusto del Noce, El suicidio de la revolución (1978). Otra corriente, con la que me identifico, reclama la autonomía de la tradición marxista y encuentra sus raíces en la obra de Antonio Labriola, que remite en este tema a Marx (por ejemplo, Frosini, 2010; Bianchi, 2008; Thomas, 2009). A partir de su posición marxista-labrioliana, Gramsci absorbe y "metaboliza” para sí lo que considera conveniente para su propio proyecto intelectual y politico del pensamiento de Croce y Gentile, ambos discípulos directos de Labriola. Sobre la relación Gramsci-Labriola, ver Fresu, 2016. 
La expresión "filosofía de la praxis" aparece por primera vez en el Cuaderno n. 5, $\$ 127$, donde se refiriere a Maquiavelo como autor de libros "de acción política inmediata", en cuya "crítica del presente", habrían expresado:

[...] una concepción del mundo original, que podría también ella llamarse "filosofía de la praxis" o "neo-humanismo" en cuanto que no reconoce elementos trascendentales o inmanentes (en sentido metafísico), sino que se basa toda ella en la acción concreta del hombre que por sus necesidades históricas actúa y transforma la realidad (cc 5, \$127, t. II, p. 342; Q 657, itálicos meus).

La dimensión del desafío teórico lanzado por este pequeño pasaje es enorme.

Para una aproximación adecuada al concepto de praxis considero conveniente acudir a la discusión gramsciana del concepto de "creatividad", planteada por Marx en su primera tesis sobre Feuerbach (la cuestión del "lado activo" de la subjetividad humana en la aprehensión de la realidad) y su crítica a la concepción pasiva del pensamiento humano. En este sentido, indica:

\footnotetext{
Para escapar al solipsismo y al mismo tiempo a las concepciones mecanicistas que están implícitas en la concepción del pensamiento como actividad receptiva y ordenadora, hay que plantear la cuestión "historicistamente" y al mismo tiempo poner en la base de la filosofía la “voluntad" (en último análisis la actividad práctica o política). (cc 11, \$59, t. IV, p. 331; Q 1486).
}

Si hasta la filosofía clásica alemana, la filosofía fue concebida como "una actividad receptiva o a lo sumo ordenadora, o sea, que fue concebida como conocimiento de un mecanismo que funcionaba objetivamente fuera del hombre", la filosofía clásica alemana "introdujo el concepto de 'creatividad' del pensamiento", sin embargo, en un "sentido idealista y especulativo" (Idem, ibidem). Siendo así, afirma Gramsci:

Parece que sólo la filosofía de la praxis hizo dar un paso adelante al pensamiento, sobre la base de la filosofía clásica alemana, evitando toda tendencia al solipsismo, historizando el pensamiento en cuanto que lo asume como concepción del mundo, como "buen sentido" difundido en el gran número (Idem, Ibidem).

4. En las referencias a los Cuadernos de la cárcel, remitiremos a la edición crítica publicada por la editorial Era, indicada como cc seguido de los números de cuaderno, parágrafo y páginas respectivos. Acompañaremos esta referencia con la indicación de la misma cita en la edición crítica en lengua italiana, indicada como $\mathrm{Q}$, seguida del n. de página. 
Y en este sentido, la noción de creatividad, en su vínculo con la praxis política, asume un nuevo significado: “creativo hay que entenderlo en el sentido 'relativo', de pensamiento que modifica el modo de sentir del mayor número y por lo tanto de la realidad misma que no puede ser pensada sin este mayor número" (Idem, ibidem), en evidente relación con el concepto de hegemonía en arduo proceso de elaboración en esos días de la cárcel. Y finalmente, en una dirección fundamental para el tema que nos ocupa:

Creativo también en el sentido de que enseña que no existe una "realidad" válida por símisma, en sí y por sí, sino en relación histórica con los hombres que la modifican, etcétera (Idem, ibidem, itálicos meus).

Reténgase este fundamental posicionamiento que será crucial en el tratamiento de los temas que abordaremos en lo que sigue.

\subsection{Devenir}

La posición teórica de Gramsci puede ser descripta en una de sus dimensiones como una teoría del devenir, una efectiva filosofía del movimiento: una kineología propongo llamarla sintéticamente, en contraste explícito con aquel modo de concebir el mundo y su dinámica que se ha denominado ontología, aunque evitando cualquier tentación de remisión a una "primera filosofía”. Veamos un ejemplo clave de este modo de pensamiento en el tratamiento de un tema fundamental planteado por Marx en la sexta tesis sobre Feuerbach:

Que la "naturaleza humana” es el "conjunto de las relaciones sociales" es la respuesta más satisfactoria, porque incluye la idea del devenir: el hombre deviene, se transforma continuamente con el transformarse de las relaciones sociales [...]. Puede incluso decirse que la naturaleza del hombre es la "historia" (y en este sentido, postulando historia = espíritu, que la naturaleza del hombre es el espíritu) si precisamente se da a la historia el significado de "devenir" (CC 7, §35, t. III, pp. 172/73; Q 884).

Y en una dirección central para el tema foco de este trabajo: "el hombre es un devenir. También el conocimiento es un devenir, por lo tanto, también la objetividad es un devenir" (CC 8, § 177, t. III, p. 307; Q 1049).

Reviste particular interés en este punto mostrar la forma en que Gramsci piensa la compleja relación entre práctica bumana y conocimiento reflexionando sobre una actividad que permite vivenciar la radical historicidad del mundo: el "trabajo" ("la actividad teórico-práctica del hombre”), como principio educativo, 
[... crea los primeros elementos de una intuición del mundo, liberada de toda magia y brujería, y da la base al desarrollo ulterior de una concepción histórica, dialéctica, del mundo, para comprender el movimiento y el devenir, para valorar la suma de esfuerzos y de sacrificios que ha costado el presente al pasado y que el futuro cuesta al presente, para concebir la actualidad como síntesis del pasado, de todas las generaciones pasadas, que se proyecta en el futuro (CC 12 , 2 , t. IV, p. 374-375, Q 1541).

Para finalizar este tópico, quisiera evidenciar el hecho de que, en esta concepción centrada en la historicidad, la acción política - y la "ciencia" enlazada con ella -, es vista como una praxis que debe captar el mundo in fieri, en su movimiento complejo del "hacerse", para promover su transformación:

Para el hombre político toda imagen "fijada" a priori es reaccionaria: el político considera todo el movimiento en su devenir [...]. El político imagina al hombre como es y al mismo tiempo como debería ser para alcanzar un determinado fin; su trabajo consiste precisamente en conducir a los hombres a moverse, a salir de su ser presente para volverse capaces colectivamente de alcanzar el fin propuesto, o sea a “conformarse” al fin. (cC 15, \$58, t. v, pp. 230-231; Q 1820).

Las dos categorías presentadas aquí sucinta y esquemáticamente nos brindan el marco conceptual apropiado para introducir el concepto que es el foco principal de este texto.

\subsection{Objetividad}

Gramsci elabora su posición en relación con la cuestión de la objetividad en el contexto de la crítica demoledora al "manual” de Nicolai Bujarin, tomado como modelo de un modo "degradado" del desarrollo del materialismo histórico y, por lo tanto, modelo a ser sometido a un meticuloso proceso crítico. Como señala Haug (2001, p. 70) “de lo que Gramsci se dio cuenta fue que la fuente vital del pensamiento marxista había sido olvidada o hecha a un lado por el propio marxismo"s.

Si bien el trabajo erosivo de la elaboración gramsciana_tiene diversos focos, su crítica a la concepción bujariniana de objetividad es central y articula una serie

5. Como consecuencia de esto, continua Haug (2001, p. 73), “La extra histórica, supuestamente eterna verdad, fue el 'materialismo dialéctico', por entonces canonizado. Según la ideología oficial, su aplicación a la historia y la sociedad daría lugar al materialismo histórico. Sin embargo, Gramsci, que a este respecto pensaba exactamente en la línea de Marx, entendió que la presunción de un primero, absoluto conocimiento es un absoluto antihistóricismo. Gramsci responde a esta auto-renuncia del marxismo con la tesis del historicismo absoluto". 
compleja de temas. Veamos, en primer lugar, la cuestión referente a la existencia del "mundo externo". Dice Gramsci:

Toda la polémica contra la concepción subjetivista de la realidad, con la cuestión "terrible" de la "realidad objetiva del mundo externo", está mal planteada, peor conducida y en gran parte es fútil y ociosa [...]. Elpúblico popular no cree ni siquiera que se pueda plantear semejante problema, de si el mundo externo existe objetivamente. Basta enunciar asi el problema para sentir un irrefrenable y pantagruélico estallido de hilaridad. El público "cree" que el mundo externo es objetivamente real... (cc 11.\$17, t. IV, p. 273; Q 1411, itálicos meus).

Encontramos en estas líneas un primer movimiento fundamental del autor de los Cuadernos en la dirección de la construcción de su posición, constituido de la siguiente manera: (a) en primer lugar en el modo mordaz con el que se refiere al dramatismo del problema surgido por su planteo equivocado: la "terrible cuestión"; (b) en segundo lugar, en el uso de la figura de la risa del público popular (del sentido común, dirá en otra parte) como recurso retórico para resaltar el modo "fútil y ocioso" del tratamiento del tema por parte de Bujarin, que es fútil y ocioso porque no se encuentra allí el problema: sobre la existencia del mundo exterior no se puede argumentar cientificamente. Destacar este movimiento teórico es fundamental porque, inmediatamente, comienza su segundo paso: el trabajo crítico sobre esta risa.

"Pero aquí nace precisamente la cuestión" afirma Gramsci, y se pregunta metodológicamente: ‘cuál es el origen de esta creencia [del público popular en el mundo externo] y qué valor crítico tiene objetivamente' ?" Porque, "si el 'sentido común' se burla, el filósofo de la praxis debería igualmente buscar una explicación tanto del significado real que tiene la concepción, como de por qué la misma ha nacido y se ha difundido entre los intelectuales, y también de por qué hace reír al sentido común” (CC 11, \$17, t. IV, pp. 276-277; Q 1412-1413). Y responde:

De hecho esta creencia es de origen religioso [...]. Puesto que todas las religiones han enseñado y enseñan que el mundo, la naturaleza, el universo ha sido creado por dios antes de la creación del hombre y por lo tanto el hombre ha encontrado el mundo ya listo y acabado, catalogado y definido de una vez por todas (cc 11, \$17, t. IV, p. 273; Q 1411-1412).

En este sentido, el Ensayo popular debería ser criticado por "haber acogido la concepción de la realidad objetiva del mundo externo en su forma más trivial y acrítica” (Ibidem). Vistas mejor las cosas,

No es tan fácil justificar un punto de vista de objetividad exterior tan mecánicamente enten- 
dida. ¿Parece que pueda existir una objetividad extra-histórica y extrahumana? ¿Pero quién juzgará sobre tal objetividad? ¿Quién podrá ponerse en esta especie de "punto de vista del cosmos en sí” y qué significará semejante punto de vista? Puede perfectamente sostenerse que se trata de un residuo del concepto de dios, precisamente en su concepción mística de un dios ignoto (cc, loc. cit., p. 276; Q 1415).

Enfoquemos con más nitidez la crítica gramsciana sobre esta noción metafísica de objetividad que se recicla, "en forma ingenua”, en la concepción expresa en el materialismo de Bujarin:

El concepto de "objetivo" del materialismo metafísico parece que quiere significar una objetividad que existe fuera del hombre; pero cuando se afirma que una realidad existiría aún si no existiera el hombre, se hace una metáfora o se cae en una forma de misticismo (cC 11, t. IV, pp. 276-277; Q 1416).

Pero entonces, ¿ por dónde debería comenzar un tratamiento adecuado del problema desde de una posición que escape de la metafísica, sea la del idealismo o la del materialismo mecanicista? Veamos la orientación metodológica que establece el comunista sardo remitiéndose a los fundadores de la tradición marxista. Por un lado, remitiéndose a Engels en su tesis según la cual "la unidad del mundo consiste en su materialidad demostrada [...] por el largo y laborioso desarrollo de la filosofía y las ciencias naturales" contiene precisamente el germen de la concepción justa, porque se recurre a la historia y al hombre para demostrar la realidad objetiva" (Idem, ibidem, itálicos meus).

Por el otro lado, buscando apoyo en una de las posiciones teóricas de Marx más utilizadas en los Cuadernos afirma:

El problema de la "objetividad" del conocimiento según la filosofía de la praxis, puede ser elaborado partiendo de la proposición [...] de que "los hombres adquieren conciencia (del conflicto entre las fuerzas materiales de producción), en el terreno ideológico", de las formas jurídicas, políticas, religiosas, artísticas, filosóficas. ¿Pero esta conciencia se limita al conflicto entre las fuerzas materiales de producción y las relaciones de producción - según la letra del texto -, o se refiere a todo conocimiento consciente? Este es el punto que es preciso elaborar y que puede serlo con todo el conjunto de la doctrina sobre el valor de las superestructuras (Idem, ibidem).

Partiendo de estas orientaciones, y articulándolas con los ejes teórico-metodológicos de las Tesis sobre Feuerbach, Gramsci establece su propia y polémica definición 
(dentro del marxismo y de los propios estudios gramscianos) de lo que debe entenderse por "objetividad" en el marco de la filosofía de la praxis. Esbozada inicialmente de forma dubitativa en su primera redacción en el Cuaderno $8, \$ 177$, es resuelta de la siguiente manera en la segunda escritura en el Cuaderno 11, \$17:

Objetivo quiere decir siempre "humanamente objetivo", lo que puede corresponder en forma exacta a "históricamente subjetivo". O sea: que objetivo significaría "universalmente subjetivo". El hombre conoce objetivamente en cuanto el conocimiento es real para todo el género humano históricamente unificado en un sistema cultural unitario; pero este proceso de unificación histórica se produce con la desaparición de las contradicciones internas que desgarran la sociedad humana, contradicciones que son la condición de la formación de los grupos y del nacimiento de las ideologías no universales concretas sino hechas caducas inmediatamente por el origen práctico de su sustancia. Hay pues una lucha por la objetividad (para liberarse de las ideologías parciales y falaces) y esta lucha es la misma lucha para la unificación cultural del género humano. Lo que los idealistas llaman "espíritu" no es un punto de partida, sino de llegada, el conjunto de las superestructuras en devenir hacia la unificación concreta y objetivamente universal y no ya un presupuesto unitario, etcétera (cc $11, \S 17$, t. IV, pp. 276-277; Q 1415/16, itálicos meus).

Destaco este pasaje que considero absolutamente central: "hay una lucha por la objetividad"; lucha entre posiciones, interpretaciones, concepciones de mundo, que construyen imágenes diversas de lo que sean el universo, la especie humana y la sociedad, y en este sentido, se puede percibir como "objetividad" se enlaza con hegemonía.

Una de las cuestiones cruciales involucradas en estas discusiones es la polémica cuestión de la relación de estas posiciones gramscianas con el "materialismo", razón por la cual destaco brevemente a continuación algunos elementos de su reflexión sobre el asunto.

Del materialismo histórico a la filosofía de la praxis.

\subsection{Materia, materialismo}

\subsubsection{Materia}

Un tema correlato a la crítica del concepto metafísico de objetividad en el manual de Bujarin, es la crítica del concepto de materia y, consecuentemente de materialismo, contenido en este texto fundante del marxismo soviético. En estos puntos la posición gramsciana es tan polémica como lo es su definición de objetividad. En primer lugar, delimita los contendientes: 
Es evidente que para la filosofía de la praxis la "materia” no debe ser entendida ni en el significado que se desprende de las ciencias naturales (física, química, mecánica, etc.) ni en sus significados tales como se desprenden de las diversas metafísicas materialistas (cC 11, t. IV, p. 297; Q 1441).

En segundo lugar, criticando lo que llama hipóstasis ("es decir abstracción arbitraria”, cc, 11, \$32, p. 301; Q 1447) del concepto de “materia”, examina la cuestión de las diversas propiedades físicas atribuidas a la materia en el proceso histórico del conocimiento humano. En este tema, Gramsci recurre a diversos ejemplos uno de los cuales vale la pena traer al texto: la "cosa" y el concepto de "fuerza mecánica del vapor".

¿El conjunto de las propiedades de todo tipo de material ha sido alguna vez el mismo? La historia de las ciencias técnicas demuestra que no. ¿Durante cuánto tiempo no se hizo caso de la fuerza mecánica del vapor? ¿Y puede decirse que tal fuerza mecánica existiera antes de ser utilizada por las máquinas humanas? Entonces, ¿en qué sentido y hasta qué punto no es cierto que la naturaleza no da lugar a descubrimientos e inventos de fuerzas preexistentes, de cualidades preexistentes de la materia, sino sólo a "creaciones" que están estrechamente ligadas a los intereses de la sociedad, al desarrollo y a las ulteriores necesidades de desarrollo de las fuerzas productivas? (cC 11, \$30, t. 4, pp. 297-298; Q 1442/43).

Y concluye observando que, en la filosofía de la praxis,

Las diversas propiedades físicas [...] de la materia <que> en su conjunto constituyen la materia misma (a menos que no se recaiga en una concepción del nóumeno kantiano) son consideradas, pero sólo en cuanto resultan 'elemento económico' productivo. La materia, pues, no debe ser considerada como tal, sino como social e históricamente organizada para la producción (Idem, ibidem).

De este modo, aquello que deba ser entendido por "materia" no es "dado", es una construcción histórica y en devenir.

\subsubsection{Materialismo}

La cuestión del "posicionamiento filosófico” de Gramsci es tal vez el problema más difícil de procesar en el marco de los estudios gramscianos, y por esto posiblemente el más polémico dentro del propio campo, como advertimos anteriormente. En esta área temática era dominante la influencia de la posición de Engels (1987, pp. 361362) sobre lo que éste denominó "el problema cardinal” o "supremo" de la filosofía: la oposición entre materialismo e idealismo, entendido como "principios": 
El gran problema cardinal de toda la filosofía, especialmente de la moderna, es el problema [...] de la relación entre el pensar y el ser, entre el espíritu y la naturaleza, problema supremo de toda la filosofía [...] Los filósofos se dividían en dos grandes campos, según la contestación que diesen a esta pregunta. Los que afirmaban el carácter primario del espíritu frente a la naturaleza $[. .$.$] formaban en el campo del idealismo. Los otros, los que reputaban la naturaleza$ como lo primario, figuran en las diversas escuelas del materialismo.

El texto de Engels es un momento central en la construcción de lo que Haug (2001, p. 74) denomina el "decisionismo"6 de carácter metafísico, que se instala en el materialismo histórico contra Marx en su volverse hacia la metafísica en busca de un principio. Gramsci se rebela contra esta posición, y esto, según el filósofo alemán, "no lo alejaba de Marx sino que, por el contrario, lo llevaba a comenzar de nuevo desde el propio punto de partida de Marx" (Haug, 2001, p. 71).

En razón de esta posición crítica, Giuseppe Prestipino (2009, p. 516) sugiere que "no parece que se aplique a Gramsci la calificación de materialista (al menos sin ulteriores especificaciones), una calificación más o menos adecuada, en cambio, para la mayor parte de los marxistas". Veamos más cuidadosamente esta "ulteriores especificaciones" en la construcción del posicionamiento gramsciano sobre el materialismo.

Deberíamos comenzar el punto subrayando que, para el pensador sardo_la cuestión de las "relaciones entre estructura y superestructura" puede ser considerada como "el problema crucial del materialismo histórico” (cC4, \$37, t. II, p. 167; Q 455) y en este sentido remite a Marx, señalando que un "punto de referencia para comprender las relaciones entre estructura y superestructuras se halla contenido en Miseria de la filosofía" obra entendida como "momento esencial en la formación de la filosofía de la praxis" y que "puede ser considerada como el desarrollo de las Tesis sobre Feuerbach” (CC 13, \$18, t. v, p. 42; Q 1592) 7 .

6. En su crítica a Luciene Sève en torno de "la notoria 'cuestión fundamental de la filosofía" sobre la que el filósofo marxista francés "exige de Gramsci [...] un reconocimiento directo de la primacía ontológica de la materia”, Haug pone en evidencia la aporía fundamenta que atraviesa lo que el autor denomina sugestivamente como "decisionismo ontológico". Gramsci parece percibir que la "resolución materialista de la cuestión fundamental de la filosofia” es, de hecho, una decisión que, ipso facto, pone una conciencia o un sujeto en una posición decisiva en el doble sentido de la palabra. Al final, la "firme afirmación" de Sève de la primacía de la materia es, a pesar de su aparente decisión y firmeza, un acto subjetivo. Lo no-primario reconoce lo primario, pero la primacía de este acto descansa en la posición del renunciante porque éste renuncia a sí mismo - y lo hace pero sin saber que lo hace). Este es el decisionismo de la teoría ontológica del reflejo. La autodeclaración de materialismo filosófico está así plagada por la dialéctica irónica de que carga consigo un aspecto tanto del subjetivismo como del idealismo. Esta es la insalvable paradoja que necesariamente acecha a la "tomada de posición materialista" o decisión ontológica.

7. Sobre la evolución del tratamiento gramsciano de la dupla estructura/superestructura, remito al libro de 
En este sentido puede señalarse que el punto de partida de Gramsci es el punto de arribo de Marx en su crítica a la distinción metafísica entre "materia" y "espíritu". Ciertamente hay una inocultable valorización de los resultados del "idealismo", en un modo desprejuiciado de pensar que es heredado de la tradición marxiana, la cual se colocaba, no lo olvidemos, como heredera del punto más alto de la filosofía clásica alemana. En este sentido, indica, "Hegel [...] dialectizó los dos momentos de la vida filosófica, materialismo y espiritualismo". Sus seguidores, al contrario, "destruyeron esta unidad" y se regresó, por un lado, "al viejo materialismo con Feuerbach" y, por el otro "al espiritualismo de la derecha hegeliana". En su juventud, Marx habría "revivido" esta experiencia, reconstituyendo una unidad superior: hegeliano primero, materialista feuerbachiano después, en su devenir marxista reconstituye "la unidad destruida en una nueva construcción filosófica: ya en las tesis sobre Feuerbach aparece nítidamente esta su nueva construcción, esta su nueva filosofía” (cc 4, $\$ 3$, t. 2, p. 136; Q 424); estas nuevas posiciones "demuestran precisamente hasta qué punto superó Marx la posición filosófica del materialismo vulgar” (cc 5, §39, t. II, p. 274; Q 572). En términos de la evolución de la filosofía de la praxis,

En el pasaje sobre el "materialismo francés en el siglo XIII" (Sagrada familia) está bastante bien y claramente mencionada la génesis de la filosofía de la praxis: ésta es el "materialismo" perfeccionado por la labor de la misma filosofía especulativa y fundido con el humanismo. Es verdad que con estos perfeccionamientos del viejo materialismo queda sólo el realismo filosófico (cc 11, \$16, t. IV, p. 271; Q 1408, itálicos meus).

Si "del viejo materialismo queda sólo el realismo filosófico”, ¿Cómo debería ser planteada en este marco la cuestión del "principio", del "problema fundamental", como lo denomina Engels en el texto que citamos $?^{8}$ No debería plantearse de ese modo; en cambio, se pregunta Gramsci: “¿Qué significará, [...] el término 'monismo’”? y responde inmediatamente, escapando ciertamente del decisionismo metafisico:

Giuseppe Cospito El ritmo del pensamiento de Gramsci (2016), posiblemente el más meticuloso trabajo filológico realizado hasta el presente en éste y otros tópicos.

8. Giuseppe Cospito (2010, p. 302) indica que "los Cuadernos parten de esta dicotomía, que aparece en el subtítulo de los 'Apuntes de filosofía, pero la superan en la elaboración completa de la filosofía de la praxis". Gramsci señala de forma aguda la fuente engelsiana de algunos de los problemas del ensayo de Bujarin; por ejemplo, en un texto de única redacción de 1933 afirma que "El origen de muchos despropósitos contenidos en el Ensayo debe buscarse en el Antidühring en el intento, demasiado exterior y formal, de elaborar un sistema de conceptos en torno al núcleo original de filosofía de la praxis, que satisficiese la necesidad escolástica de plenitud” (cC 15, $\$ 31$, t. v, p. 203; Q 1786. Traducción parcialmente corregida por el autor, itálicos meus). 
Ciertamente no el materialista ni el idealista, sino identidad de los contrarios en el acto histórico concreto, o sea actividad humana (historia-espíritu) en concreto ${ }^{9}$, conectada indisolublemente a una cierta "materia" organizada (historicizada), a la naturaleza transformada por el hombre. Filosofía del acto (praxis, desarrollo), pero no del acto "puro", sino precisamente del acto "impuro", real en el sentido más profano y mundano de la palabra (cC 11, \$64, t. IV, p. 337; Q 1492).

Esta posición crítica es fundamental en la definición de los parámetros del tránsito del materialismo histórico para la filosofía de la praxis. Y es central también en un segundo sentido en la medida que señala, en su distinción entre el "acto puro" y el "acto impuro, profano", la radical oposición al actualismo gentiliano en el marco de un tema (la cuestión del devenir) que superficialmente puede parecer de similar abordaje.

Así, afirma el autor de los Cuadernos, la dialectización entre materialismo y espiritualismo que intentó Hegel, realmente la logró Marx y en este sentido lamenta el hecho de que "muchos materialistas históricos han repetido con Marx lo que ya antes se hizo con Hegel, esto es, de la unidad dialéctica han regresado al materialismo crudo” (cc 4, \$3, t. II, p. 136; Q 424) ${ }^{10}$. Y en la misma dirección, alerta:

Habrá que señalar cómo Marx siempre evita llamar "materialista” a su concepción y cómo cada vez que habla de filosofías materialistas las critica o afirma que son criticables. Además, Marx no emplea nunca la fórmula "dialéctica materialista" sino "racional" en contraposición a "mística", lo cual da al término "racional" un significado bien preciso (cC 8, \$206, t. III, p. 321; Q 1065).

Desde este punto de vista, propone: "habría que escribir un nuevo Anti-Dühring, que podría ser un Anti-Croce, porque en él podría resumirse no sólo la polémica contra la filosofía especulativa, sino también, implícitamente, la polémica contra el positivismo y las teorías mecanicistas, deterioro de la filosofía de la praxis" (cc 8, $\$ 235$, t. III, p. 343; Q 1088).

9. En su primer redacción de este texto (cc 4, \$37, t. II, p. 167; Q 455) escribía? “Ni el monismo materialista ni el idealista, ni 'Materia’ ni 'Espíritu’ evidentemente, sino 'materialismo histórico', o sea actividad del hombre (historia) en concreto.... En el pasaje de la primera para la segunda redacción se evidencia la supresión del término "materialismo histórico".

10. En su segunda redacción insiste Gramsci "El desgarramiento sufrido por el hegelianismo se ha repetido para la filosofía de la praxis, esto es, de la unidad dialéctica se ha regresado por una parte al materialismo filosófico, mientras que la alta cultura moderna idealista ha tratado de incorporar aquello que de la filosofía de la praxis le era indispensable" (CC 16, §9, p. 264; Q 1861). 
En realidad, este trabajo que dice Gramsci "habría que escribir" es el que él mismo realiza en gran parte en sus Cuadernos sin llegar, por razones obvias, a percibir la dimensión de la obra.

Estos pasajes son reveladores del lugar y relevancia de la batalla teórica desplegada en los Cuadernos contra lo que llama "corriente decadente" en el marxismo, aquella que le restó capacidad hegemónica; y parece ser obsesivo en esta batalla el anclaje teórico en la primera tesis sobre Feuerbach, que Gramsci desarrolla en los cuadernos hasta sus últimas consecuencias. Si, como afirma Marx en esta tesis, "el defecto fundamental de todo el materialismo anterior -incluido el de Feuerbach- es que sólo concibe las cosas, la realidad, la sensoriedad, bajo la forma de objeto o de contemplación, pero no como actividad sensorial humana, no como práctica, no de un modo subjetivo" y que, en consecuencia, "el lado activo fuese desarrollado por el idealismo, por oposición al materialismo" (aunque "sólo de un modo abstracto, ya que el idealismo, naturalmente, no conoce la actividad real, sensorial, como tal"), Gramsci desarrolla una lucha implacable en el marco de este campo abierto por Marx. Este acento radical en la praxis, sumado al efecto de la enconada lucha contra las tendencias deterioradoras del materialismo (vulgar, fatalista, mecanicista, economicista, determinista, entre otras denominaciones) lo conduce a rescatar ciertas dimensiones de las corrientes idealistas, lo que le es achacado, - al mismo tiempo que incomprendido en su radical fundamento marxiano -, por una parte de los teóricos gramscianos; por ejemplo, en Brasil, en la pluma de Carlos Nelson Coutinho, con su crítica a la posición gramsciana sobre el tema que estamos abordando, como posición idealista, sin más ${ }^{11}$.

\subsubsection{Materialismo e idealismo en Gramsci (sub. 3)}

La convicción gramsciana de que el marxismo, como corriente cultural y política, debería disputar la hegemonía tanto en el campo del sentido común y de las ideologías populares como en el campo de la "alta cultura", lo lleva a una lucha en varios frentes. En el primer campo de luchas, siendo fundamental la batalla por la conciencia popular, el enemigo principal no parece ser el "idealismo" ("la filosofía que tiene su punto culminante con Hegel"), sino la religión en tanto fuente de supercherías, y todo tipo de visiones místicas del mundo, estructuradoras de un sentido común que debería ser desmantelado y reconstruido por la nueva concepción de mundo. En este

11. Ver, por ejemplo, Coutinho, (1981 y 2011). Siendo imposible por razones de espacio en este texto, en un próximo trabajo espero poder exponer algunas discusiones brasileras en torno de esta crítica de Coutinho y extenderme sobre mi propia opinión sobre el asunto. 
sentido, evalúa positivamente el papel desempeñado por las corrientes idealistas, dado que "las teorías idealistas son el mayor intento de reforma moral ${ }^{12} \mathrm{e}$ intelectual que se haya verificado en la historia para eliminar la religión del campo de la civilización" (cC 8, \$215, t. III, p. 331; Q 1076). Por lo tanto, no es de ningún modo paradojal que la consideración de Gramsci por la cual "la concepción de las superestructuras en el materialismo histórico" ["los hombres se vuelven conscientes (de este conflicto) en el terreno ideológico' de las formas jurídicas, políticas, religiosas, artísticas o filosóficas" (cc 4, §37, t. II, p. 167; Q 455)] pueda ser considerada deudora, en cierta medida, del idealismo, como no lo fue que el hecho Marx haya tenido que recurrir al idealismo hegeliano en su crítica del materialismo metafísico. Mario Tronti (apud Haug, 2000, p. 10), llega a sugerir que Gramsci se encontró en la situación de "tener que redescubrir el marxismo a través de las lentes del idealismo"13.

En este marco crítico, puede entenderse la fuertísima reacción gramsciana frente al libro de Bujarin entendido como un compendio del materialismo vulgar "incrustado" en el marxismo. Es un "idealismo al revés", señala agudamente:

La filosofía del Ensayo popular (implícita en él) puede ser llamada un aristotelismo positivista, una adaptación de la lógica formal a los métodos de las ciencias físicas y naturales. [...] Si el "idealismo especulativo" es la ciencia de las categorías y de la síntesis a priori del espíritu, o sea una forma de abstracción antihistoricista, la filosofía implícita en el Ensayo popular es un idealismo al revés, en el sentido de que conceptos y clasificaciones empíricas sustituyen a las categorías especulativas, tan abstractas y antihistóricas como éstas (cC 11, \$14 t. IV, pp. 266-267; Q 1402-1403).

De este modo, señalando el carácter relacional de la filosofía de la praxis, alerta para el hecho de que, en contraste con el idealismo - que "hipostasia [...], la calidad, hace de ella un ente en sí mismo, el 'espíritu”, del mismo modo que la religión "hizo de ella la divinidad" -, en ésta "la calidad está siempre vinculada a la cantidad" destacando aún que "incluso seguramente en tales conexiones está su parte más original y fecunda", y en este sentido advierte:

Pero si es hipóstasis la de la religión y la del idealismo, o sea abstracción arbitraria y no proceso de distinción analítica prácticamente necesario por razones pedagógicas, es también

12. La edición en español de los cuadernos, en un claro descuido traduce "riforma morale" por "reforma laboral" en este trecho de los Cuadernos, razón por la cual he debido corregir la cita.

13. Según la opinión de Haug (Ibidem), Tronti habría ido "lejos demás" en esta evaluación. El texto de Mario Tronti citado por Haug se encuentra en "Tra materialismo dialettico e filosofia della prassi", En: Caracciolo/Scalia, La città futura, 1959, p. 88. 
hipóstasis la del materialismo vulgar, que diviniza una materia hipostática (CC 11, \$32, t. IV, p. 301 ERA, Q 1447).

Y delinea con precisión el proceso de dogmatización que se haría la marca de ese marxismo degradado:

Por eso sucede también que la misma filosofía de la praxis tiende a convertirse en una ideología en el peor sentido, o sea un sistema dogmático de verdades absolutas y eternas; especialmente cuando, como en el Ensayo popular, aquella es confundida con el materialismo vulgar, con la metafísica de la "materia" que no puede ser sino eterna y absoluta (cc $11 \$ 62$, t. IV, pp. 334-335; Q 1489).

Afectada de esta radical incomprensión de la originalidad de la posición de Marx, en la corriente criticada "en realidad se reproduce aún la posición recíprocamente unilateral criticada en la primera tesis sobre Feuerbach entre materialismo e idealismo" y se torna necesario "al igual que entonces, si bien en un momento superior", operar nuevamente "la síntesis en un momento de desarrollo superior de la filosofía de la praxis" (cc $10 \$ 10$, t. IV, p. 145, Q 1248-49), tarea que asume como desafío en los Cuadernos, y en la cual ciertamente alcanza fundamentales avances que contribuyeron con la renovación contemporánea de la herencia de Marx.

Ciertamente hay en Gramsci una crítica durísima a las formas vulgarizadas (que se alarga hacia otras más sofisticadas) del materialismo; sin embargo, considero que hay que forzar mucho el espíritu de sus escritos para tornarlo "idealista" en este plano de reflexiones, como sugieren ciertas lecturas, aunque sus escritos puedan ser torturados en una u otra dirección para que digan lo que el intérprete - incluyendo el que aquí escribe- quiere decir. En otros términos, sobre los textos de Gramsci se descarga la misma batalla por su "objetividad" que el autor señalaba para cualquier objetividad.

Es perfectamente comprensible, en razón de los argumentos anteriores, el esfuerzo por distinguir su posición tanto del idealismo cuanto del materialismo mecanicista, ambos especulativos, "metafísicos" y en este sentido manifieste la tendencia a llamar a su propia posición como filosofía de la praxis. Ahora, es claramente incorrecta la interpretación que hace de este esfuerzo de delimitación con las formas mecanicistas del materialismo un abandono de la posición materialista como tradición cultural en la lucha por la interpretación del mundo. La crítica gramsciana a la corriente degradada debe ser cuidadosamente distinguida de una caida en el idealismo.

Si la intervención gramsciana significa rechazar el materialismo en su forma de "primera filosofía", como decisión que se toma especulativamente esto implica el desplazamiento a un nuevo terreno, el de una radicalmente nueva posición teórica, 
el terreno de un "nuevo materialismo", podemos decir si necesitásemos satisfacer nuestra propia "necesidad escolástica de plenitud” como la llamaba Gramsci en su crítica a Engels (Q. 15, \$31, p. 1786) y si sobre esa expresión se aplica la carga crítica que estamos discutiendo; uno que conciba "las cosas, la realidad, la sensoriedad" como praxis, desarrollando "el lado activo" de la subjetividad humana: "materialismo histórico”, si el acento se coloca en el segundo término como advierte Gramsci o, posiblemente más apropiado, "materialismo práctico", o "de la praxis", como sugiere Haug (2001, p. 74).

En todo caso, no se trataría de abandonar el materialismo como tradición cultural sino de "traducir" la problemática materialista al lenguaje de la filosofía de la praxis, colocándola por entero en el plano de la praxis humana: un historicismo radical, “integral” (Q. 26, \$5, p. 2298) o “absoluto" (Q. 15, \$61, p. 1826) en las palabras de Gramsci.

En este sentido, el "materialismo práctico" como traducción de las viejas posiciones materialistas-metafísicas en el ámbito de la filosofía de la praxis, es "materialismo" en un sentido infinitamente superior a la metafísica de la materia en la que incurrió el marxismo de la segunda y tercera internacional.

\subsection{Materialismo histórico}

El centro del esfuerzo teórico de Gramsci puede ser visto como una tentativa colosal de revitalizar el marxismo, de volver a dotarlo de la capacidad expansiva perdida en las complejas redes de las luchas históricas. En este sentido, con base en lo tratado en la sección anterior, intentaré exponer sucintamente su "diagnóstico" sobre el materialismo histórico y los caminos sugeridos para su actualización.

En primer lugar, observa que, aunque en su consideración el materialismo histórico pueda ser visto como "la coronación de todo este movimiento de reforma intelectual y moral, en su dialéctica cultura popular-alta cultura”, éste "atraviesa aún la fase popular, se ha convertido incluso en 'prejuicio' y 'superstición'. El materialismo histórico, tal como es, es el aspecto popular del historicismo moderno" (cC 4 $\$ 3$, t. II, p. 134; Q 424), señalando en este proceso de difusión y popularización, un fenómeno que denomina de "doble combinación” y "doble revisión”,

[...] la filosofía de la praxis ha sufrido realmente una doble revisión, o sea que ha sido subsumida en una doble combinación filosófica. Por una parte, algunos de sus elementos, de manera explícita o implícita, han sido absorbidos e incorporados por algunas corrientes idealistas (basta citar a Croce, Gentile, Sorel, el mismo Bergson, [el pragmatismo]; por la otra, los llamados ortodoxos, preocupados por hallar una filosofía que fuese, según su punto 
de vista muy restringido, más amplia que una "simple" interpretación de la historia, creyeron ser ortodoxos, identificándola fundamentalmente con el materialismo tradicional. Otra corriente ha vuelto al kantismo (CC 16, \$9, t. IV, p. 259; Q 1854/55) $)^{14}$.

Por otra parte, señala que "en el período moderno de la historia el materialismo histórico" éste se encuentra más difundido de lo que parece, "y sin embargo, se presenta bajo el aspecto de 'economismo histórico”" (cC 4 \$38, t. II, p. 174; Q 462), y habiendo degenerado en economicismo histórico,

[...] el materialismo histórico pierde gran parte de su expansividad cultural entre las personas inteligentes, tanta como adquiere entre los intelectuales perezosos, entre aquellos que quieren parecer siempre sagacísimos, etcétera; esto, como escribió Engels, hace creer a muchos que pueden tener, a poco precio y sin ninguna fatiga, toda la historia y toda la sabiduría política en el bolsillo (cc, loc. cit., 175-176; Q 463).

Por lo tanto, es necesario estar atentos para el hecho de que "también el materialismo histórico tiende a convertirse en una ideología en el sentido peyorativo, o sea en una verdad absoluta y eterna" (cc 4, \$40, t. II, p. 178; Q 466), es decir que "en el materialismo histórico se ha formado una corriente decadente” (cC 7, §1, t. III, p. 143; Q 851) y observa,

Así como el catolicismo popular puede ser traducido en los términos del "paganismo" o de otras religiones correspondientes, el materialismo histórico decadente puede ser traducido a los términos "teológicos", o sea de la filosofía prekantiana y precartesiana (CC 7, §1, t. III, p. 143; Q 851).

Lo curioso del caso, constata Gramsci con una dramática actualidad ${ }^{15}$, es que "a menudo sucede que se combate al economismo histórico creyendo combatir al materialismo histórico” (cC $4 \$ 38$, t. II, p. 175; Q 462).

Y se indaga el prisionero “' por qué la filosofía de la praxis ha tenido este destino, de haber servido para formar combinaciones, con sus elementos principales,

14. En la primer redacción se lee: “por el otro, los marxistas 'oficiales', preocupados por encontrar una 'filosofía' que contuviese al marxismo, la han hallado en las derivaciones modernas del materialismo filosófico vulgar o incluso en corrientes idealistas como el kantismo (Max Adler)”. (cc 4, \$3, t. II, pp. 133-134; Q 421-422).

15. Digo esto en el sentido de que es sin duda un hecho absurdo que, 80 años después de estas reflexiones, en las salas y discusiones universitarias y mucho más en ámbito de la llamada “opinión pública”, aún tengamos que soportar y combatir la asociación del marxismo con estas visiones degradadas que ya fueron tanto tiempo atrás refutadas y superadas. 
tanto con el idealismo como con el materialismo filosófico?”. Poniendo de relieve las dificultades para dar una respuesta adecuada alerta que "el trabajo de investigación no puede ser sino complejo y delicado: exige mucha delicadeza en el análisis y sobriedad intelectual":

La identificación de los conceptos que la filosofía de la praxis ha "cedido" a las filosofías tradicionales y por los que éstas han encontrado algún instante de rejuvenecimiento, debe hacerse con mucha cautela crítica, y significa ni más ni menos que hacer la historia de la cultura moderna después de la actividad de los fundadores de la filosofía de la praxis (cc 16 $\$ 9$, t. v, p. 260; Q 1856).

Y hacia el interior del propio campo marxista, se pregunta “ ¿Por qué incluso los llamados ortodoxos han 'combinado' la filosofía de la praxis con otras filosofías y con una predominantemente más que con otra?” Este aspecto de la cuestión "es aún más interesante", destaca, observando que, sin embargo, "de hecho, la que cuenta es la combinación con el materialismo tradicional", ya que "la combinación con el kantismo no ha tenido más que un éxito limitado y sólo entre grupos intelectuales restringidos" (cC $16 \$ 9$, t. v, p. 261; Q 1857). "La que cuenta es la combinación con el materialismo tradicional", este es nuestro punto focal no sólo porque sobre esta combinación el autor de los Cuadernos se ve obligado a descargar el peso de su crítica, sino también porque esta combinación ha perdurado en el tiempo y nos desafía todavía hoy. Como un avance de respuesta a estas preguntas podrían ser consideradas las siguientes observaciones, fundamentales para el tema:

La filosofía de la praxis tenía dos tareas: combatir las ideologías modernas en su forma más refinada para poder constituir su propio grupo de intelectuales independientes, y educar a las masas populares, cuya cultura era medieval. Esta segunda tarea, que era fundamental dado el carácter de la nueva filosofía, absorbió todas sus fuerzas no sólo cuantitativamente, sino también cualitativamente; por razones "didácticas", la nueva filosofía se ha combinado en una forma de cultura que era un poco superior a la media popular (que era muy baja), pero absolutamente inadecuada para combatir las ideologías de las clases cultas, mientras que la nueva filosofía había nacido precisamente para superar la más alta manifestación cultural de la época, la filosofía clásica alemana, y para suscitar un grupo de intelectuales propios del nuevo grupo social del cual era la concepción del mundo (cc 16, $\$ 9$ TV, p 262; Q 1857-58).

A pesar del peso y la extensión de las corrientes criticadas Gramsci encuentra en el trabajo de Antonio Labriola una contracorriente solitaria y un estímulo al trabajo crítico: 
Labriola se distingue de unos y otros con su afirmación de que el marxismo es una filosofía independiente y original. En este sentido hay que trabajar, continuando y desarrollando la posición de Labriola. La tarea es muy compleja y delicada (CC 4, \$3, t. II, pp. 133/34; Q 421-422).

A partir de su tesis de la autosuficiencia del marxismo, es decir, de la afirmación de “que la filosofía del marxismo está contenida en el marxismo mismo", Labriola será visto por Gramsci como "el único que trató de construir científicamente la filosofía de la praxis" (cc $11 \$ 70$, t. IV, pp. 348/49; Q 1507/08).

Lo que percibe es que, en su camino de transformación en una filosofía de masas, el instrumental teórico del materialismo histórico había perdido vigor heurístico y capacidad hegemónica, haciéndose necesario un arduo combate por su renovación. Este es un punto central de la intervención gramsciana no siempre tenido suficientemente en cuenta: se trataba (y se trata aún) de recuperar la posición de la tradición teórico política con origen en Marx no sólo como "horizonte filosófico insuperable de nuestro tiempo" según la expresión de Jean-Paul Sartre en razón de que "las circunstancias que lo han engendrado no han sido superadas", sino para potenciar su capacidad de dialogar productivamente y atraer para su esfera de argumentación teórica al pensamiento avanzado de nuestro tiempo.

De este modo, en Gramsci el concepto del “materialismo histórico” va tomando distancia del materialismo metafísico, del positivismo, del reduccionismo y del fatalismo histórico y en torno de esa crítica construye una concepción de historia donde ninguna objetividad "natural" se impone, donde es vital la acción humana en permanente lucha por la interpretación de esos mismos hechos que la praxis humana construye, lucha en la cual ese material histórico se hace historia pensada, una determinada objetividad, siempre en riesgo de una nueva reabsorción interpretativa, de una nueva hegemonía. Sin embargo, en esta renovación radical la tradición comenzaba a cargar un nombre, no por casualidad asociado a la figura de Antonio Labriola.

\subsection{Filosofía de la praxis}

Quisiera introducir esta sección trayendo al texto un pasaje de los Cuadernos donde se resumen una buena cantidad de los aspectos cruciales discutidos hasta aquí: se trata de las nociones de "Oriente” y "Occidente”. No por acaso en el título que Gramsci le dio al parágrafo 25 del Cuaderno 7 (cc, t. 3, p. 163; Q 874) se lee: "Objetividad de lo real” y en su segunda redacción en el parágrafo 20 del Cuaderno 11 (cC, t. 4, p. 279; Q 1419) “Objetividad y realidad del mundo externo". En el mismo indica: 
Para entender exactamente los significados que puede tener el problema de la realidad del mundo externo, puede ser oportuno desarrollar el ejemplo de las nociones de "Oriente" y "Occidente" que no dejan de ser "objetivamente reales", si bien ante el análisis demuestran no ser otra cosa que una "construcción” convencional o sea "histórico-cultural” (cc 11, §25, t. IV pp. 279/80; Q 1419).

Inmediatamente respondiendo a su propia indagación sobre “¿Qué significaría Norte-Sur, Este-Oeste sin el hombre?”, nos brinda un conjunto de reflexiones fundamentales para el tema que nos ocupa:

\begin{abstract}
Éstas son relaciones reales y sin embargo no existirían sin el hombre y sin el desarrollo de la civilización. Es evidente que Este y Oeste son construcciones arbitrarias, convencionales, o sea históricas, porque fuera de la historia real cada punto de la tierra es Este y Oeste al mismo tiempo. Esto se puede ver más claramente en el hecho de que estos términos han cristalizado no desde el punto de vista de un hipotético y melancólico hombre en general, sino desde el punto de vista de las clases cultas europeas que a través de su hegemonía mundial los han hecho aceptar dondequiera. El Japón es Extremo Oriente no sólo para el europeo sino seguramente también para el norteamericano de California y para el mismo japonés, el cual a través de la cultura política inglesa podrá llamar Cercano Oriente a Egipto. Así, a través del contenido histórico que se ha ido aglutinando al término geográfico, las expresiones Oriente y Occidente han acabado por indicar determinadas relaciones entre complejos de civilizaciones distintas (cC, idem, ibidem, Q 1419/20).
\end{abstract}

Y sin embargo, a pesar de los términos "no ser otra cosa que una 'construcción' convencional o sea 'histórico-cultural", se trata de referencias "reales":

Corresponden a hechos reales, permiten viajar por tierra y por mar y llegar exactamente a donde se había decidido llegar, permiten "prever" el futuro, objetivar la realidad, comprender la objetividad del mundo externo. Racional y real se identifican. Parece que sin haber comprendido esta relación no se puede comprender la filosofía de la praxis, su posición frente al idealismo y el materialismo mecánico, la importancia y el significado de la doctrina de las superestructuras (Idem, Q 1420).

¿Está Gramsci cometiendo un crimen de leso idealismo al recuperar el aforismo hegeliano? Su respuesta no puede ser más clara: lejos del idealismo, pero también del materialismo metafísico, está construyendo una piedra fundamental de la filosofía de la praxis. 
No es exacto que en la filosofía de la praxis la "idea" hegeliana haya sido sustituida por el "concepto" de estructura, como afirma Croce. La "idea" hegeliana es resuelta tanto en la estructura como en las superestructuras y todo el modo de concebir la filosofía ha sido "historizado", o sea que se ha iniciado el nacimiento de un nuevo modo de filosofar más concreto e histórico que el precedente (Idem, ibidem).

Poco después del inicio de la circulación de la edición temática de los Cuadernos, donde el grupo editor coordenado por Felice Platone, bajo la supervisión de Palmiro Togliatti, difundía su hipótesis sobre el uso de la expresión Filosofía de la Praxis que atribuían a la necesidad del prisionero de eludir la censura carcelaria [la tesis del “camuflaje”, le dirá Haug (2000, p. 5), o el "mito" de las “palabras en código" según la crítica de Green (2011, p. 388)], Rodolfo Mondolfo en su clásico texto "En torno a Gramsci y la filosofía de la praxis", (edición italiana de 1955), apunta, por primera vez hasta donde me consta, en una dirección opuesta:

La preferencia que manifiestan los cuadernos de Gramsci por la expresión "filosofía de la praxis", en lugar de "materialismo histórico", no se debe tanto, como para otras expresiones, a la necesidad de eludir las sospechas de la censura carcelaria (sobre la cual llama la atención el editor de sus notas), como al hecho de que la segunda expresión estaba "demasiado ligada a una concepción determinista inferior del marxismo” (Mondolfo, 1986, p. 215).

Esta doble matriz de interpretación se extiende hasta nuestros días. Sin embargo, hay indicaciones de que la posición inaugurada por Mondolfo ha tendido a imponerse entre los estudiosos del filósofo de la praxis sardo [Dainoto (2009); Haug (2000); Frosini (2002, 2010); Thomas (2015); Vacca (2016a, 2016b) etc.]. Es que, en su evolución degradante, la interpretación criticada habría descuidado el hecho de que en la expresión "materialismo histórico" era necesario "poner el acento en el segundo término ‘histórico’ y no en el primero de origen metafísico” (cC $11 \$ 27$, t. IV, p. 293, Q 1437), obscureciendo el hecho de que:

La filosofía de la praxis es el "historicismo" absoluto, la mundanización y terrenalidad absoluta del pensamiento, un humanismo absoluto de la historia. En esta línea hay que excavar el filón de la nueva concepción del mundo (Idem, ibidem).

En esta nota Gramsci coloca otra de los pilares fundamentales de la filosofía de la praxis. En efecto, el "historicismo absoluto", en conjunto con la batería de conceptos que expusimos, fundamentalmente en su concepción del devenir, nos brinda un código de lectura a partir del cual se puede recuperar el filón más original y produc- 
tivo del paradigma teórico creado por Marx y el más capaz de recuperar el diálogo creativo y propulsor con la línea de frente de la cultura contemporánea.

Por otra parte, es fundamental no perder de vista el modo en que en Gramsci, el criterio historicista es llevado al tratamiento de la efectividad y caducidad de las filosofías, criterio que alcanza, obviamente, a la filosofía de la praxis. Dado que "según el concepto más amplio de historicidad de la filosofía [...] una filosofía es 'histórica' en cuanto se difunde, en cuanto se vuelve concepción de la realidad de una masa social (con una ética correspondiente)” (Q. $10 \$ 31$, t. IV, pp. 163-164; Q 1270-71):

De ello se deduce también que el carácter de la filosofía de la praxis es especialmente el de ser una concepción de masas, una cultura de masas y de masas que operan unitariamente, o sea que tienen normas de conducta no sólo universales en idea, sino "generalizadas" en la realidad social (Idem, ibidem).

Provocativamente, Fabio Frosini (2010) la llamará, en la misma dirección de estas proposiciones gramscianas, "la religión del hombre moderno".

Además de la cuestión de su efectividad histórica, Gramscillama la atención para la historicidad, es decir, el movimiento, el devenir y la caducidad, indicando que "como filosofía el materialismo histórico afirma que cada 'verdad' creída eterna y absoluta tiene orígenes prácticos y ha representado o representa un valor provisional”, siendo esto válido también para la filosofía de la praxis. Ahora, si bien esto es fácilmente aceptable para las otras filosofías, "lo difícil es hacer comprender 'prácticamente' esta interpretación por lo que respecta al materialismo histórico mismo"16 (Q. $4 \$ 40, \mathrm{t}$. II, pp. 177-178; Q 465).

En función de esta radical originalidad que hemos intentando explicitar, se comprende que no es exagerada la expresión de Peter Thomas $(2015,104)$ que califica la elaboración teórica realizada en los Cuadernos de la Cárcel como una verdadera "refundación” de la tradición centrada en el nombre de Marx.

16. Es importante tener en cuenta ese "prácticamente" que Gramsci coloca entre comillas seguramente para subrayar el hecho de que "teóricamente" es posible que la idea de una historicidad y caducidad sea aceptada, pero "prácticamente" las cosas no son tan fáciles. Ocurre algo similar con la cuestión de economicismo: difícilmente se encuentre una forma de marxismo que "teóricamente" se autodefina como "economicista", sin embargo, "prácticamente" la historia de la tradición es bastante más poblada de este tipo de lecturas. 
Referências Bibliográficas

Bianchi, Alvaro. (2008), O laboratório de Gramsci: filosofia, história e politica. São Paulo, Alameda.

BuJARIN, Nicolai. ([1921] 1970), Tratado de materialismo histórico [Esp. Teoría del materialismo histórico: ensayo popular de sociología marxista]. Rio de Janeiro, Laemmert.

Coutinho, Carlos Nelson. (1981), Gramsci. Porto Alegre, L\&pm.

Coutinho, Carlos Nelson. (2011), De Rousseau a Gramsci: ensaios de teoria politica. São Paulo, Boitempo.

Cospito, Giuseppe. (2010), “O marxismo soviético e Engels: o problema da ciência no caderno 11”. In: Aggio, Alberto et al. (orgs.). Gramsci no seu tempo. Brasília/Rio de Janeiro, Fundação Astrojildo Pereira/Contraponto.

Cospito, Giuseppe. ([2011] 2016), El ritmo del pensamiento de Gramsci: una lectura diacrónica de los Cuadernos de la Cárcel. Trad. Juan Jorge Barbero. Ciudad Autónoma de Buenos Aires, Continente.

Dainotto, Roberto. (2009), "Filosofía della Praxis”. In: Dizionario gramsciano 1926-1937. Roma, Carocci.

Del Noce, Augusto. (1978), Il suicidio della rivoluzione. Milão, Rusconi.

ENGELs, Friedrich. (1987), “Ludwig Feuerbach y el fin de la filosofía clásica alemana”. In: MARX, Karl \& Engels, Friedrich. Obras escogidas. Buenos Aires, Cartago, t. 4.

Fresu, Gianni. (mar. 2016), “Labriola, Gramsci e o materialismo histórico italiano”. Revista Outubro, 25.

Frosini, Fabio. (2002), "La 'filosofia della praxis’ nei Quaderni del Carcere di A. Gramsci”. Sonomia. Rivista di Filosofia.

Frosini, Fabio. (2010), La religione dell'uomo moderno. Roma, Carocci.

Gramsci, Antonio. (1981-2001), Cuadernos de la cárcel. Puebla, Era, tomo I a VI.

Gramsci, Antonio. (1977), Quaderni del Carcere. Edición crítica del Instituto Gramsci. Org. Valentino Gerratana. Turim, Einaudi.

Green, Marcus. (2011), "Rethinking the subaltern and the question of censorship in Gramsci's Prison Notebooks”. Postcolonial Studies, 14 (4): 387-404.

Haug, Wolfgang Fritz. (2000), "Gramsci's 'philosophy of praxis". Socialism and Democracy, 14 (1), 1-19.

HaUG, Wolfgang Fritz. (2001), "From Marx to Gramsci, from Gramsci to Marx: historical materialism and the philosophy of praxis". Rethinking Marxism, 13 (1): 69-82.

Labriola, Antonio. (s/d), Filosofia y socialismo: consideraciones sobre filosofía, politica del proletariado, economía, historia, etc., desde el punto de vista marxista. Buenos Aires, Claridad.

Lenin, Vladimir I. (1975), Cuadernos filosóficos. Obras completas, tomo XLII. México, Akal Editor.

Lenin, Vladimir I. (1990), Materialismo y empiriocriticismo. La Habana, Pueblo y Educación. 
Liguori, Guido \& Voza, Pasquale (eds.). (2009), Dizionario gramsciano. Roma, Carocci. Marx, Karl. (2011), O capital. São Paulo, Boitempo.

Marx, Karl. (1987), Tesis sobre Feuerbach. Obras escogidas, tomo 1. Buenos Aires, Cartago.

Mondolfo, Rodolfo. (1986), "En torno a Gramsci y la filosofía de la praxis". In: Marx y el marxismo. México, Fondo de Cultura Económica.

Prestipino, Giuseppe. (2009), "Materialismo e materialismo volgare”. In: Dizionario gramsciano 1926-1937. Roma, Carocci, pp. 593-594.

Thomas, Peter D. (2009), The Gramscian moment: philosophy, hegemony and marxism. Leiden, Brill.

Thomas, Peter D. (2015), “Gramsci’s marxism: the philosophy of praxis". In: McNaLly, Marc (ed.). Antonio Gramsci. Basingstoke, Palgrave Macmillan.

VACCA, Giuseppe. (2016a), “Dal materialismo storico alla filosofia della praxis”. International Gramsci Journal, 2 (1): 358-377.

VACCA, Giuseppe. (2016), Modernidades alternativas: o século XX de Antonio Gramsci. Brasília, Fundação Astrojildo Pereira.

\section{Resumen}

El concepto de objetividad en Gramsci

El trabajo discute el tratamiento de la cuestión de la objetividad realizada por Gramsci en los Cuadernos de la cárcel, sugiriendo inicialmente la existencia de dos polos de tensiones teóricas en el interior de la tradición marxista hasta entonces: las Tesis sobre Feuerbach y su crítica al materialismo metafísico y el tipo de materialismo filosófico que desagua en el marxismo soviético. A partir de esta posición inicial, en la primera parte del artículo son discutidos los conceptos de praxis, "lado activo" del pensamiento humano, devenir y objetividad. En la segunda parte es abordado, en primer lugar, el posicionamiento sobre el concepto de materia y el materialismo filosófico, subrayando la crítica gramsciana al deterioro del marxismo por la búsqueda metafísica de una "primera filosofía" y la batalla por reintegrarlo a la perspectiva marxista de la praxis, sin por esto adoptar una posición idealista; en segundo lugar, se muestra cómo, recuperando la idea labrioliana de autosuficiencia del marxismo, Gramsci erosiona hasta tal punto las bases del materialismo histórico en la forma criticada que la propia tradición debe ser denominada ya con otro nombre: filosofía de la praxis.

Palabras-clave: Gramsci; Objetividad; Filosofía de la Praxis. 


\section{Abstract}

\section{The concept of objectivity in Gramsci}

The paper discusses Gramsci's treatment of the objectivity issue in the Prison Notebooks, initially suggesting the existence of two poles of theoretical tension within the Marxist tradition until then: the Theses on Feuerbach and its critique of metaphysical materialism and the kind of philosophical materialism that drains into Soviet Marxism. From this initial position, in the first part of the article, the concepts of praxis, "active side" of human thought, becoming and objectivity are discussed. In the second part is addressed, first, the positioning on the concept of matter and philosophical materialism, underlining Gramscian criticism of the deterioration of Marxism by the metaphysical search for a "first philosophy" and the battle to reintegrate it into the Marxist perspective of praxis, without thereby adopting an idealist position; secondly, it shows how, recovering the Labriolian idea of Marxism's self-sufficiency, Gramsci erodes to such an extent the bases of historical materialism in the criticized form that the tradition itself must be called by another name: philosophy of praxis.

Keywords: Gramsci; Objectivity; Philosophy of praxis.

Texto recebido em 3/5/2019 e aprovado em 6/5/2019.

DOI: $10.11606 / 0103-2070 . t s .2019 .157686$

Raúl Burgos é professor titular no Departamento de Sociologia e Ciência Política da Universidade Federal de Santa Catarina. E-mail: raul.ufsc@gmail.com. 